\title{
El vínculo con los perros y con los gatos durante el estado de alarma por la pandemia de COVID-19 en España
}

\author{
Jaume Fatjó, LV, PhD, Dipl.ECAWBM-BM ${ }^{1,2}$ \\ Elena García, LV, MSc. ${ }^{1}$ \\ Patricia Darder, LV, MSc. ${ }^{1}$ \\ Juan Argüelles, LV. ${ }^{3}$ \\ Jonathan Bowen, BVetMed, MRCVS, DipAS(CABC) ${ }^{1,4}$
}

1. Cátedra Fundación Affinity Animales y Salud. Departamento de Psiquiatría y Medicina Legal. Facultad de Medicina. Universidad Autónoma de Barcelona. 08193 - Bellaterra (Barcelona).

2. IMIM-Mar Health Park, Neuropsychiatry and Drug Addiction Institute (INAD), Barcelona, Spain.

3. Cardenal Herrera-CEU University, Alfara del Patriarca, Valencia, Spain

4. Queen Mother Hospital for Small Animals, Royal Veterinary College, North Mymms, Hertfordshire, UK.

Cita recomendada. FATJÓ, J., GARCÍA, E., DARDER, P., ARGÜELLES, J., BOWEN, J., El vínculo con los perros y con los gatos durante el estado de alarma por la pandemia de COVID-19 en España, dA. Derecho Animal (Forum of Animal Law Studies) 11/4 (2020). - DOI https://doi.org/10.5565/rev/da.544

\section{Resumen}

El presente texto tiene como objetivo discutir los resultados de un estudio previo llevado a cabo sobre los efectos del estado de alarma por la COVID-19 en España sobre las personas, sus perros y sus gatos. Ayuda a comprender el apoyo que la gente obtiene de sus animales, sobre todo en momentos difíciles, y cómo el vínculo con los animales puede sustituir algunos aspectos de las relaciones humanas que podrían verse comprometidos durante una pandemia, como la compañía y el contacto físico. También se valora el efecto del confinamiento en los centros de acogida de animales de compañía. Estos resultados pueden ayudar a desarrollar intervenciones sanitarias que aprovechen el valor como capital social de la convivencia con animales de compañía.

Palabras clave: COVID-19; Vínculo humano-animal; Antrozoología; Comportamiento canino; Comportamiento felino.

\section{Abstract - The bond with dogs and cats during the COVID-19 pandemic state of alarm in Spain}

This paper aims to discuss the results of a previous study that looked at the effects of the COVID-19 confinement on people and their dogs and cats. It helps us to understand the support that people get from their animals, particularly at difficult times, and how the human-animal bond can compensate for certain aspects of human relationships that become compromised during a pandemic, such as companionship and physical contact. It also evaluates the effect of confinement on Spanish Animal Shelters. Our findings suggest ways in which public health interventions can take advantage of pets as a valuable source of social capital.

Key words: COVID-19; Human-animal bond; Anthrozoology; Canine behavior; Feline behavior. 


\section{Introducción}

La pandemia de COVID-19 ha afectado de forma muy negativa la calidad de vida de la población. A los efectos directos de la enfermedad sobre la salud se suman, por un lado, el impacto sobre el estilo de vida del confinamiento y, por otro, la ansiedad derivada de un futuro incierto, tanto por lo que se refiere a la economía como al equilibrio social. En este sentido, una revisión sistemática reciente indica que la pandemia ha empeorado de forma significativa los indicadores de salud mental de la población en numerosos países, incluido España ${ }^{1}$.

Desde el decreto del primer estado de alarma en marzo de 2020, los animales de compañía han sido objeto de la atención del público y de los medios de comunicación por 4 motivos principales. En primer lugar, por su papel como miembros de la red social de la persona. En segundo lugar, por los efectos que las restricciones han podido tener sobre su comportamiento y bienestar. En tercer lugar, por el posible aumento de abandonos y reducción de la adopción de animales abandonados derivados de la crisis económica y social. Por último, por la posibilidad de que los animales sean infectados por el virus SARS-CoV-2 y puedan actuar como vectores de transmisión de la enfermedad.

El presente trabajo se centra en los efectos beneficiosos que ha supuesto y supone la convivencia con perros y con gatos durante la pandemia de COVID-19, así como en el impacto que el confinamiento y la restricción de la movilidad social parecen tener sobre su adaptación y calidad de vida. El presente trabajo pretende ser un resumen ampliado de la conferencia realizada por los autores sobre este tema en el Webinar Internacional Coronavirus y Animales organizado por ICALP del 22 de junio al 16 de julio de 2020. Tal y como se indicó durante la intervención, los datos completos sobre el impacto de la pandemia durante el primer estado de alarma en España fueron publicados en junio de forma preliminar y en noviembre de forma definitiva en el Journal of Veterinary Behavior².

En una primera parte, presentaremos el modelo teórico que utilizamos para evaluar la relación que establecen las personas con sus perros y con sus gatos. En la segunda parte, presentaremos de forma resumida los resultados de nuestro estudio durante el período de confinamiento derivado del primer estado de alarma. En la tercera parte, valoraremos el impacto del confinamiento en los centros de acogida para animales.

\section{La medición del vínculo con los perros y con los gatos}

La relación que se establece entre una persona y su animal de compañía es única e irrepetible. Sin embargo, a lo largo de los últimos años se han desarrollado modelos teóricos y herramientas psicométricas que permiten capturar y cuantificar aquellas dimensiones comunes a cualquier relación entre una persona y su perro o su gato. Desde hace aproximadamente 10 años, nuestro grupo de investigación se ha centrado en la aplicación del modelo del intercambio social al análisis de las relaciones que se establecen entre las personas y los animales.

La teoría del intercambio social establece que cualquier relación puede ser valorada en términos del balance entre los aspectos positivos y negativos que supone la convivencia para cada uno de sus integrantes ${ }^{3}$. Cuando los elementos positivos superan a los negativos podemos decir que la relación goza de buena salud. Por el contrario, cuando los aspectos negativos igualan o superan a los positivos la relación entraría en un área de riesgo, que podría desembocar en su ruptura. Hace unos años el modelo del intercambio social fue aplicado al estudio de las relaciones entre las personas y los perros. La investigación realizada resultó en el desarrollo y validación de una escala psicométrica denominada MDORS (Monash Dog Owner Relationship Scale). La escala MDORS evalúa tres dimensiones básicas de la relación: el patrón de interacción, la cercanía emocional y el denominado coste percibido asociado a la convivencia ${ }^{4}$. La dimensión de interacción valora la frecuencia e intensidad de las actividades que la persona realiza junto a su animal de compañía. La cercanía emocional trata de describir el grado de apoyo que la persona recibe de su perro, sobre todo en momentos de dificultad. El coste percibido estima el impacto negativo que la presencia del animal tiene en la calidad de vida de la

1.XIONG J, LIPSITZ O, NASRI F, LUI LMW, GILL H, PHAN L, CHEN-LI D, IACOBUCCI M, HO R, MAJEED A, MCINTYRE RS. Impact of COVID-19 pandemic on mental health in the general population: A systematic review. J Affect Disord, 277 (2020) 55-64.

2.BOWEN J, GARCIA E, DARDER P, ARGÜELLES J, FATJÓ J. The effects of the Spanish COVID-19 lockdown on people, their pets, and the human-animal bond. J Vet Behav (2020) Available at: https://doi.org/10.1016/j.jveb.2020.05.013

3.EMERSON RM. Social Exchange Theory. Annu Revi Sociol, 2 (1976) 335-362.

4.DWYER F, BENNETT PC, COLEMAN GJ. Development of the Monash Dog Owner Relationship Scale (MDORS). Anthrozoos, $19 / 3$ (2006) 243-256. 
persona, tanto por lo que se refiere a los aspectos económicos como, sobre todo, a los relacionados con el estilo de vida. Por ejemplo, la presencia del perro puede limitar o poner barreras a la realización de ciertas actividades por parte de la familia, como podrían ser los viajes o el acceso a espacios donde no se admiten perros. En definitiva, de acuerdo con el modelo, cualquier relación entre una persona y su perro podría representarse como un punto en un espacio tridimensional, definido por 3 ejes que representarían las 3 dimensiones que acabamos de describir.

En el año 2016 colaboramos con el grupo de investigadores australianos que en su día crearon la escala MDORS, para desarrollar una adaptación de esta herramienta a las relaciones entre las personas y los gatos ${ }^{5}$. La escala CORS (Cat Owner Relationship Scale) parte de las mismas premisas y cuenta con la misma estructura que su homónima MDORS, con las modificaciones necesarias para evaluar el patrón de interacción propio de las relaciones entre las personas y los gatos.

\section{La convivencia con animales durante el primer estado de alarma en España}

Coincidiendo con estado de alarma aplicado el 14 de marzo de 2020, pusimos en marcha un estudio para evaluar el impacto de la pandemia tanto en las personas como en sus animales de compañía ${ }^{6}$.

A través de su difusión en diversas redes sociales pudimos capturar una muestra de conveniencia formada por 1297 propietarios de perros y de gatos de toda España. Cada participante nos proporcionó información detallada sobre cómo la pandemia estaba afectando a su calidad de vida, al comportamiento de su animal de compañía y a la relación que les unía a ambos, a través de las escalas MDORS y CORS que hemos descrito en el apartado anterior. El $82 \%$ de los participantes en el estudio había estado confinado durante un período de entre 3 y 4 semanas en el momento de proporcionar la información.

Tres de cada cuatro participantes en el estudio nos indicaron que su perro o su gato había sido una fuente importante de apoyo durante el estado de alarma, más que durante el período previo a la pandemia. Si seguimos el modelo del intercambio social citado anteriormente, durante el confinamiento las personas aumentaron la interacción con sus perros y con sus gatos, se sintieron más ligadas a ellos emocionalmente, y se redujo el coste percibido de la relación.

El apoyo social es uno de factores más importantes de protección de la salud mental y el bienestar de la persona. Una buena fuente de apoyo social es aquella que está siempre disponible, resulta cercana desde un punto de vista emocional y ofrece la posibilidad de contacto físico ${ }^{7}$. Los animales de compañía satisfacen casi a la perfección estas tres demandas y, por ello, son tan valorados por la gente como integrantes de la red social. En relación con el patrón de interacción, la inmensa mayoría de participantes aumentó el contacto físico con sus animales durante el confinamiento. Los acariciaron, los besaron y, en el caso de los perros, los abrazaron más que antes. Este hallazgo nos parece particularmente interesante. Por un lado, pone en valor la convivencia con animales de compañía en un momento en que el contacto físico entre personas se ve limitado por las políticas sanitarias. En este sentido, convivir con un animal podría ser para muchas personas una forma de sobrellevar mejor las restricciones de movilidad y de contacto social, al disponer de una válvula de escape, de alguien a quien redirigir nuestra necesidad natural de contacto físico con los demás. Por otro lado, indica que las recomendaciones de las autoridades sanitarias de mantener una cierta distancia social también con los animales de compañía, particularmente en personas positivas al COVID-19, no fueron seguidas, al menos de forma generalizada. Vemos pues que el estudio de las motivaciones subyacentes a las relaciones entre las personas y sus animales de compañía puede resultar de utilidad para diseñar acciones de comunicación más efectivas, que aumenten la adherencia de la población a las políticas de salud pública que involucran a los animales de compañía.

El valor de los perros y de los gatos como fuente de salud, de apoyo emocional y como una estrategia para reducir la sensación de soledad durante la pandemia de COVID-19 ha sido también confirmado por un estudio posterior realizado por colegas de la Universidad de Lincoln con población británica ${ }^{8}$.

En definitiva, los estudios científicos llevados a cabo durante la pandemia de COVID-19 han

5.HOWELL T, BOWEN J, FATJÓ J, et al. Development of the cat-owner relationship scale (CORS). Behav Process 14193 (2017). DOI: $10.1016 /$ j.beproc.2017.02.024

6.BOWEN J, GARCIA E, DARDER P, ARGÜELLES J, FATJÓ J. The effects of the Spanish COVID-19 lockdown on people, their pets, and the human-animal bond. J Vet Behav (2020) Available at: https://doi.org/10.1016/j.jveb.2020.05.013

7.RUSH MM. Perceived Social Support: Dimensions of Social Interaction Among Sober Female Participants in Alcoholics Anonymous. J Am Psychiatr Nurses Assoc, 8/4 (2002) 114-19; FIELD T. Touch for socioemotional and physical well-being: A review. Dev Rev, 30/4 (2010) 367-83.

8.RATSCHEN E, SHOESMITH E, SHAHAB L, SILVA K, KALE D, TONER P, et al. Human-animal relationships and interactions during the Covid-19 lockdown phase in the UK: Investigating links with mental health and loneliness. PLoS ONE, 15/9 (2020) e0239397. https://doi.org/10.1371/journal.pone.0239397 
confirmado el beneficio de convivir con animales de compañía y, por tanto, de su importancia para la sociedad y para el sistema de salud. La ayuda proporcionada por los animales de compañía es particularmente importante en momentos de dificultad y cuando, por distintos motivos, la red social de la persona se ve diezmada.

\section{El comportamiento de los perros y de los gatos durante el primer estado de alarma en España}

La información proporcionada por los participantes en el estudio nos permitió también estimar cómo las restricciones de movilidad y los cambios de estilo de vida familiar afectaron al comportamiento de los perros y de los gatos. Para interpretar de forma adecuada estos resultados es importante tener en cuenta que provienen de la descripción que los propietarios hacen del comportamiento de sus animales, y que por tanto no está libre de una cierta subjetividad.

En general podemos decir que el estado de alarma afectó al comportamiento y adaptación de una buena parte de perros y de gatos, si bien de forma distinta en ambas especies.

Los perros no vieron reducido el número de paseos diarios, pero sí su duración. Este hallazgo contrasta con la percepción popular durante el confinamiento, recogida también por los medios de comunicación, de que los perros salían a pasear más que antes del confinamiento, pues esta actividad era una de las pocas que estaba permitida en la vía pública.

Las vocalizaciones, incluidos los ladridos, los gemidos y los aullidos, aumentaron en uno de cada cuatro perros. En este sentido, fueron la dimensión del comportamiento que más empeoró durante el estado de alarma. Es interesante mencionar dos de los factores asociados a un aumento de vocalización durante el confinamiento: el tiempo total de paseo y la frecuencia con que el propietario se enfadaba con el perro. En pocas palabras, los perros que paseaban menos y entraban más en conflicto con sus propietarios vocalizaban más. La vocalización en los perros es una manifestación frecuente de frustración que, por lo tanto, podría aumentar ante una reducción de las oportunidades para realizar ejercicio y explorar el entorno. Resulta más complicado establecer una relación de causa y efecto entre el aumento de vocalizaciones y las reacciones negativas del propietario. Sea como fuere, estos resultados nos permiten confirmar el profundo impacto que tiene el entorno físico y social en el comportamiento, adaptación y bienestar del perro.

En relación con el comportamiento del gato, el confinamiento afectó sobre todo a aquellos elementos que tenían que ver con el entorno social y la interacción con los miembros de la familia. Antes hablábamos del aumento del contacto físico con los animales de compañía durante el estado de alarma y de su efecto beneficioso para la persona en términos de reducción de la percepción de estrés y de soledad. Sin embargo, es importante recordar que los gatos deben poder decidir con quien establecen contacto social, en que momento y en qué espacio lo hacen. En este sentido, una manipulación excesiva podría suponer una fuente de estrés para los gatos.

Como hemos dicho antes, la inmensa mayoría de perros y gatos cuyo comportamiento hemos descrito aquí había estado expuesto a los efectos del confinamiento durante un período no superior a 4 semanas. Serán necesarios más estudios para valorar el impacto a largo plazo de las medidas de control impuestas por la pandemia de COVID-19 en el comportamiento y bienestar de los perros y de los gatos. En relación con el perro, preocupa especialmente el aumento de problemas de comportamiento ante la separación como consecuencia de la reincorporación de los miembros de la familia al trabajo no telemático, y de los estudiantes a las aulas. En relación con los gatos, sería interesante valorar el impacto del aumento de contacto físico en su adaptación general al entorno y en su comportamiento social.

\section{Impacto del estado de alarma en los centros de acogida de animales de compañía}

El pasado mes de abril realizamos un estudio en colaboración con la Fundación Affinity para estimar el impacto del confinamiento en las protectoras de animales de compañía.

Participaron 102 centros de acogida de animales de toda España ${ }^{9}$. El número de abandonos se redujo durante el confinamiento, pero también las demandas de adopción. Sin embargo, aumentaron las peticiones de acogida temporal de perros, si bien no de gatos. Este hallazgo podría confirmar la sospecha de muchas entidades de que algunas personas podrían adoptar temporalmente a un perro para esquivar las medidas de restricción de movilidad, que permitían salir a pasear con el perro.

Las protectoras vieron reducidas las donaciones, tanto económicas como de material, así como la ayuda por parte de los voluntarios, esto último como consecuencia de las restricciones sobre la movilidad.

9.FUNDACIÓN AFFINITY. https://www.fundacion-affinity.org/blog/impacto-del-confinamiento-sobre-las-protectoras-y-laadopcion-de-animales-de-compania 


\section{Reflexiones finales}

Los datos obtenidos durante las primeras fases de la pandemia por COVID-19 en países como España y el Reino Unido confirman el valor de los perros y de los gatos como fuentes de apoyo emocional en momentos de dificultad. En ese sentido, los trabajos de investigación realizados durante los períodos de confinamiento han permitido evaluar los beneficios de la convivencia con animales en el conjunto de una población expuesta a una misma fuente de estrés y a un impacto negativo similar sobre la calidad de vida. Además, han permitido evaluar la importancia de los entornos físico y social en la adaptación y calidad de vida de los animales de compañía.

\section{Referencias}

- BOWEN J, GARCIA E, DARDER P, ARGÜELlES J, FATJÓ J. The effects of the Spanish COVID-19 lockdown on people, their pets, and the human-animal bond. J Vet Behav (2020) Available at: https://doi.org/10.1016/j.jveb.2020.05.013

- DWYER F, BENNETT PC, COLEMAN GJ. Development of the Monash Dog Owner Relationship Scale (MDORS). Anthrozoos, 19/3 (2006) 243-256.

- EMERSON RM. Social Exchange Theory. Annu Revi Sociol, 2 (1976) 335-362.

- HOWELL T, BOWEN J, FATJÓ J, et al. Development of the cat-owner relationship scale (CORS). Behav Process (2017) 14193. DOI: 10.1016/j.beproc.2017.02.024

- FIELD T. Touch for socioemotional and physical well-being: A review. Dev Rev, 30/4 (2010) 36783.

- FUNDACIÓN AFFINITY. https://www.fundacion-affinity.org/blog/impacto-del-confinamientosobre-las-protectoras-y-la-adopcion-de-animales-de-compania

- RATSCHEN E, SHOESMITH E, SHAHAB L, SILVA K, KALE D, TONER P, et al. (2020) Human-animal relationships and interactions during the Covid-19 lockdown phase in the UK: Investigating links with mental health and loneliness. PLoS ONE, 15/9 (2020) e0239397. https://doi.org/10.1371/journal.pone.0239397

- RUSH MM. Perceived Social Support: Dimensions of Social Interaction Among Sober Female Participants in Alcoholics Anonymous. J Am Psychiatr Nurses Assoc (2002) 8/4 (2002) 114-19.

- XIONG J, LIPSITZ O, NASRI F, LUI LMW, GILL H, PHAN L, CHEN-LI D, IACOBUCCI M, HO R, MAJEED A, MCINTYRE RS. Impact of COVID-19 pandemic on mental health in the general population: A systematic review. J Affect Disord, 277 (2020) 55-64. 